\title{
Bovine herpesvirus type 5 infection regulates Bax/BCL-2 ratio
}

\author{
A.F. Garcia ${ }^{1}$, J.B. Novais ${ }^{1}$, T.F. Antello ${ }^{1}$, C. Silva-Frade ${ }^{1}$, M.C. Ferrarezi ${ }^{1}$, \\ E.F. Flores ${ }^{2}$ and T.C. Cardoso ${ }^{1}$ \\ ${ }^{1}$ Laboratório de Virologia Animal e Cultivo Celular, \\ Faculdade de Medicina Veterinária, Universidade Estadual Paulista, \\ Araçatuba, SP, Brasil \\ ${ }^{2}$ Laboratório Virologia, Universidade Federal de Santa Maria, \\ Santa Maria, RS, Brasil \\ Corresponding author: T.C. Cardoso \\ E-mail: tcardoso@fmva.unesp.br \\ Genet. Mol. Res. 12 (3): 3897-3904 (2013) \\ Received October 16, 2012 \\ Accepted January 11, 2013 \\ Published September 23, 2013 \\ DOI http://dx.doi.org/10.4238/2013.September.23.8
}

\begin{abstract}
Bovine herpesvirus 5 (BoHV-5) is an $\alpha$-herpesvirus that causes neurological disease in young cattle and is also occasionally involved in reproductive disorders. Although there have been many studies of the apoptotic pathways induced by viruses belonging to the family Herpesviridae, there is little information about the intrinsic programmed cell death pathway in host-BoHV-5 interactions. We found that BoHV-5 is able to replicate in both mesenchymal and epithelial cell lines, provoking cytopathology that is characterized by cellular swelling and cell fusion. Viral antigens were detected in infected cells by immunofluorescence assay at 48 to $96 \mathrm{~h}$ postinfection (p.i.). At 48 to 72 h p.i., anti-apoptotic BCL-2 antigens were found at higher levels than Bax antigens; the latter is considered a pro-apoptotic protein. Infected cells had increased BCL-2 phenotype cells from 48 to $96 \mathrm{~h}$ p.i., based on flow cytometric analysis. At 48 to $96 \mathrm{~h}$ p.i., Bax mRNA was not expressed in any of the infected cell monolayers. In contrast, BCL-2 mRNA was found at high levels at all
\end{abstract}


p.i. in both types of cells. BoHV-5 replication apparently modulates BCL-2 expression and gene transcription, enhancing production of virus progeny.

Key words: BoHV-5; In vitro propagation; Programmed cell death; Mitochondrion

\section{INTRODUCTION}

Bovine herpesvirus type 5 (BoHV-5) belongs to the family Herpesviridae, subfamily $\alpha$-Herpesvirinae, genus Varicellovirus, and is responsible for meningoencephalitis frequently observed in young cattle and, to a lesser extent, is also incriminated in reproductive disorders. In spite of displaying different tropism and pathogenesis in vivo, in cell culture BoHV-1 and BoHV-5 share similar behavior and phenotype concerning host-cell range and cytopathic effects, mainly characterized by the production of rounded, giant cells and multinucleated syncytial cells (Marin et al., 2012).

Apoptosis, a programmed suicide cell death, characterized by chromatin condensation, DNA fragmentation, membrane blebbing and cell shrinkage, can be induced through intrinsic and extrinsic pathways (Clarke and Tyler, 2009). In the mitochondria-mediated pathway, mitochondria release several apoptotic factors, including cytochrome c, Smac/ Diablo, and apoptosis-inducing factor (AIF) into the cytosol (Ohta and Nishiyama, 2011). In addition, mitochondrial apoptotic signaling and mitochondrial outer-membrane permeabilization (MOMP) is controlled by the B-cell lymphoma 2 (BCL-2) family proteins (West et al., 2011). The anti-apoptotic BCL-2 family proteins, such as BCL-2, BCL-w, BCL-xl and myeloid cell leukemia 1 (MCL1) are generally aggregated into the outer mitochondrial membrane (West et al., 2011). Under death stimuli, Bax, another member of the BCL-2 family, displays a pro-apoptotic effect (Ohta and Nishiyama, 2011). Mammalian DNA and RNA viruses are among the stimuli that have been associated with cell apoptosis (Griffin et al., 2010). Viruses possess various biochemical and genetic mechanisms to evade and/or induce apoptosis modulation through virus-encoded proteins. Apoptosis modulation seems to be a key step in herpesvirus pathogenesis (Griffin et al., 2010). In spite of many studies on the subfamily $\alpha$-Herpesvirinae regarding anti-and-pro-apoptosis mechanisms, evidence of association of BoHV-5 with these events is completely unknown (Deruelle et al., 2010; Esaki et al., 2010; Dufour et al., 2011).

In the present study, we investigated the effect of BoHV-5 infection on BCL-2/Bax expression, and mitochondrial function in bovine Wharton's jelly-derived multipotent mesenchymal stromal cells (bWJ-MSCs).

\section{MATERIAL AND METHODS}

\section{Bovine Wharton's jelly-derived multipotent mesenchymal stromal (bWJ-MSCs)}

bWJ-MSCs were isolated and characterized as previously described, according to the Animal Care Committee at the University of São Paulo State, Brazil. The cell monolayers have been maintained in the Laboratory of Animal Virology and Cell Culture since 2010 fol- 
lowing standard procedures for seeding, freeze-thawing and propagation (Ferrari et al., 2007). The undifferentiated bWJ-MSCs at passage 30 were kept at $3.5 \times 10^{5}$ cells $/ \mathrm{mL}$ in $25-\mathrm{cm}^{2}$ tissue culture flasks (Falcon, $\mathrm{BD}$ ) at $38.5^{\circ} \mathrm{C}$ in $5 \% \mathrm{CO}_{2}$ in a humidified incubator, seeded with Advanced-DMEM (Invitrogen, Life Technologies, Carlsbad, CA, USA) with the addition of $10 \%$ fetal bovine serum (FBS; Sigma-Aldrich, St. Louis, MO, USA), 2 mM glutamine and 10 $\mathrm{mM}$ non-essential amino acids (Sigma-Aldrich) according to a standard protocol (Cardoso et al., 2007, 2012; Marin et al., 2012).

\section{Susceptibility of bWJ-MSCs cells to viral infection, viability assays and viral growth kinetics}

The BoHV-5 strain used in the infection experiment was isolated from an outbreak of neurological disease and submitted to partial sequence of the US9 gene for characterization (Ferrari et al., 2007). For infection, bWJ-MSCs were grown to $70 \%$ confluence in Lab-Tek ${ }^{\circledR}$ chamber slides (Falcon, BD) and infected with $100 \mu \mathrm{L}$ BoHV-5 suspension $\left(10^{3.2}\right.$ TCID $_{50}$ ' $\mathrm{mL}$ ), corresponding to a multiplicity of infection (m.o.i.) of 0.1 . The viral suspension was allowed to adsorb for $1 \mathrm{~h}$ at $38.5^{\circ} \mathrm{C}$. After incubation, the viral suspension was removed and the monolayers were supplemented with the same medium. Inoculated cells were monitored thereafter for cytopathic effect. Viral antigens were detected by immunofluorescence assay by reacting monoclonal anti-glyco-C viral epitopes with fixed infected and uninfected bWJ-MSCs at after infection. Cell proliferation analysis was performed using an in vitro Toxicology Assay ${ }^{\circledR}$ kit, an MTT-based assay, in both infected and uninfected bWJ-MSCs cells at 0, 24, 48, 72 and 96 h post-infection (p.i.) (TOXI-1 kit; Sigma-Aldrich). For each p.i. time, the culture supernatant was removed and $2 \mathrm{~mL}$ MTT (tetrazolium salts) added following the manufacturer recommendations (Sigma-Aldrich). Absorbance was measured at $600 \mathrm{~nm}$ with a Biophotometer (Eppendorf, Hamburg, Germany). All reported values are means of triplicate samples.

One-step growth curve of the BoHV-5 in bWJ-MSCs cells was determined in a multiplication kinetics experiment, following infection at $80 \%$ monolayer confluence at m.o.i of 1 cultured in 6-well culture plates (Falcon, BD). Adsorption was allowed for $90 \mathrm{~min}$ at $38.5^{\circ} \mathrm{C}$, the inoculum was removed, and $2 \mathrm{~mL} 2 \mathrm{X}$ fresh medium plus $1 \%$ agarose were added to determine plaque formation. The bWJ-MSCs cells were incubated at different time intervals $(24,48,72$ and $96 \mathrm{~h} \mathrm{p.i)} \mathrm{with} 1 \mathrm{~mL}$ viral suspension. At each p.i. time, the cells were fixed with $4 \%$ paraformaldehyde for $30 \mathrm{~min}$ and the agarose removed. To facilitate plaque morphology analysis and to calculate the viral titers as PFU (plaque forming units) $/ \mathrm{mL}$, all

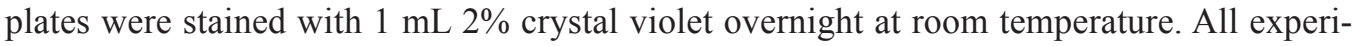
ments were performed in triplicate. Infectious virus titers were calculated according to the Spearmann-Kärber method and expressed as PFU/mL.

\section{Immunostaining for BCL-2 and Bax cell markers}

Infected and uninfected bWJ-MSCs were fixed with 4\% paraformaldehyde for $15 \mathrm{~min}$ in Lab-Tek ${ }^{\circledR}$ chamber slides (Falcon, BD). The cells were permeabilized for $10 \mathrm{~min}$ at room temperature in $0.4 \%$ Triton X-100 diluted in phosphate buffered solution (PBS). The fixed cells were incubated overnight at $4^{\circ} \mathrm{C}$ with each of the primary antibodies anti-Bax (Cat. No. 
N5413, diluted at 1:100) and anti-BCL-2 (Cat. No. SAB3300030, 1:50), both purchased from Sigma-Aldrich. On the next day, after three washes, cells were incubated with the respective secondary FITC-conjugated goat anti-mouse antibody (1:100) (Sigma-Aldrich). For nuclear staining, DAPI $(1 \mathrm{mg} / \mathrm{mL})$ was diluted in Fluormount ${ }^{\mathrm{TM}}$ aqueous medium and loaded onto samples for $15 \mathrm{~min}$. The images were collected under an AxioImager ${ }^{\circledR}$ A.1 light and an ultraviolet (UV) microscope connected to an AxioCam ${ }^{\circledR}$ MRc (Carl Zeiss, Oberkochen, Germany). The images were processed using AxioVision ${ }^{\circledR} 4.8$ software (Carl Zeiss) for each antigen, whereas values were determined by visually counting positive cells. The undifferentiated cells were submitted to the same staining procedure to assess for cross reaction among antibodies. The negative controls consisted of incubation of slides with bovine IgG and IgM isotypes as the primary antibodies.

\section{Total RNA isolation and quantitative real-time polymerase chain reaction (qPCR)}

Upon harvest of monolayer cells and respective supernatants at 24, 48, 72 and $96 \mathrm{~h}$ p.i., total RNA was extracted using Trizol $\mathrm{LS}^{\mathrm{TM}}$ protocol according to the manufacturer instructions (Invitrogen). On average, $150 \mathrm{ng}$ total RNA was used for first-strand cDNA synthesis with Enhanced Avian RT First Strand Synthesis kit (Sigma-Aldrich). qPCR was carried out and analyzed by the software on a StepOnePlus ${ }^{\circledR}$ real-time instrument (Applied Biosystems). The real-time PCR mixtures $(50 \mu \mathrm{L})$ contained $1.2 \mu \mathrm{g}$ cDNA, $400 \mathrm{nM}$ primers and $200 \mathrm{nM}$ FAM-labeled probes, customized for Bax and BCL-2 bovine sequences (Applied Biosystems). PCR was initiated by sequential amplification of 40 cycles at $95^{\circ} \mathrm{C}(15 \mathrm{~s})$ and $60^{\circ} \mathrm{C}(60 \mathrm{~s})$. The results were obtained from three replicates of each sample to ensure representative and accuracy pipetting. The expression of the bovine GAPDH (glyceraldehyde-3-phosphate dehydrogenase) gene was also quantified in a similar way for normalization. The comparative delta-delta $\mathrm{C}_{t}$ method was used to analyze the results with the expression level of the respective Bax and BCL-2 gene at the corresponding time point in infected and uninfected bWJ-MSCs in comparison to GAPDH $\mathrm{C}_{t}$ values.

\section{Statistical analysis}

All experiments were performed at least in triplicate. The results of representative experiments are presented. Descriptive statistics include the mean \pm standard deviation (s.d). $\mathrm{P}<0.001$ was considered statistically significant.

\section{RESULTS}

\section{BoHV-5 replicates in bWJ-MSCs}

BoHV-5 was able to replicate and produce cytopathic effects at $48 \mathrm{~h}$ p.i. with viral antigens detected by immunofluorescence assay (IFA) (Figure 1). The cytopathic effect was characterized as cellular swelling and cell fusion, followed by round plaques dispersed on cell monolayers (Figure 1). The intensity of viral antigens detected by IFA applying monoclonal antibody against glyco-C viral protein also increased with post-infection time. These results demonstrated that bWJ-MCS are susceptible to BoHV-5 infection. 


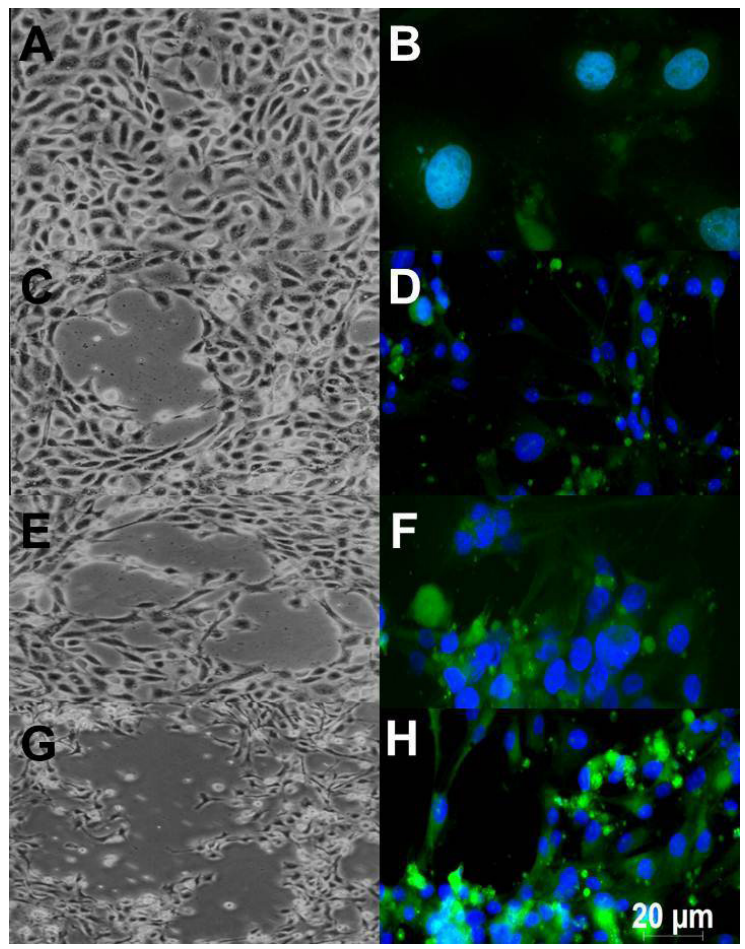

Figure 1. Photomicrographs representative of cells infected observed under phase contrast microscopy (40X magnification) and immunofluorescence for viral antigens (100X magnification). The cytopathic effect during 24 (A), $48(\mathbf{C}), 72(\mathbf{E})$, and $96(\mathbf{G}) \mathrm{h}$ post-infection (p.i.) show syncytial cells from $48 \mathrm{~h}$ p.i. The viral antigens detected by immunofluorescence assay revealed an increase of label after $48 \mathrm{~h}$ p.i. (B).

\section{BoHV-5 infection increases BCL-2/Bax ratio}

Immunostaining demonstrated Bax/BCL-2 expression at different intervals p.i. As shown in Figure 2 at 24 h p.i., infected cells did not express Bax/BCL-2 antigens on their surface, and the same was observed in uninfected cells (Figure insets). According to progressive post-infection observations, at 48 to $72 \mathrm{~h}$ p.i. higher fluorescent signals were visible for BCL-2 antigens in comparison to Bax (Figure 2). The transcription of Bax/BCL-2 genes, as ascertained by mRNA measurements, revealed that at $24 \mathrm{~h}$ p.i. Bax and BCL-2 genes were equally expressed in infected and uninfected cells, with viral titers of $3 \times 10^{3}$ $\mathrm{PFU} / \mathrm{mL}$ (Figure 3). Following an increase in virus titers (Figure 3), at 48-96 h p.i., Bax mRNA was not detected in infected cell monolayers (Figure 3). In contrast, BCL-2 mRNA was detected at p.i. times. The plaque formation revealed that BoHV-5 infection was progressive (Figure 3) when compared to uninfected bWJ-MSCs. In spite of many studies demonstrating the ability of other $\alpha$-herpesviruses to induce or suppress some pro- and anti-apoptotic factors, this is the first description of non-epithelial cells expressing higher BCL-2 amounts during infection, accompanied by Bax suppression during the productive phase of viral replication. 


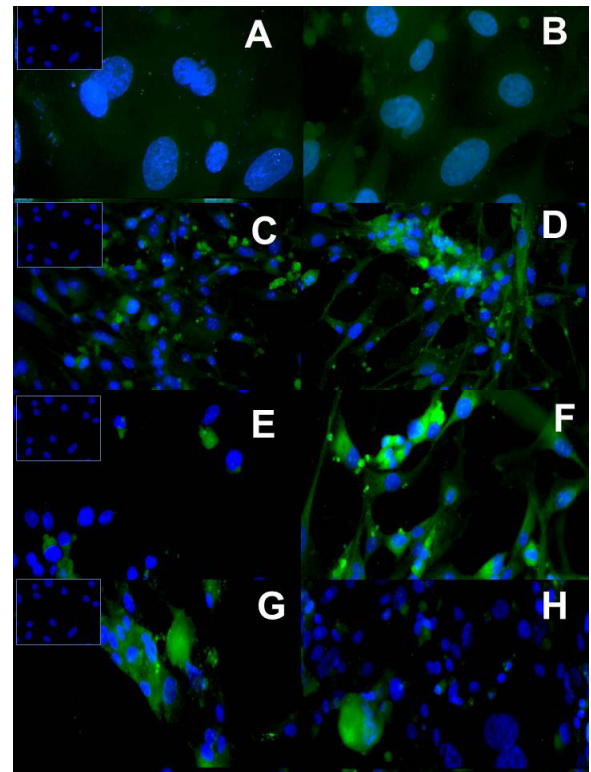

Figure 2. Immunofluorescence images showing the Bax and BCL-2 antigens observed under fluorescence microscopy (100X magnification). The intensity of Bax antigens detection was observed at higher grade at 48 and $96 \mathrm{~h}$ post-infection (p.i) (C-G). BCL-2 antigens were detected mostly at 48 and $72 \mathrm{~h}$ p.i. (D-F). The inset images show the uninfected bWJ-MSCs.
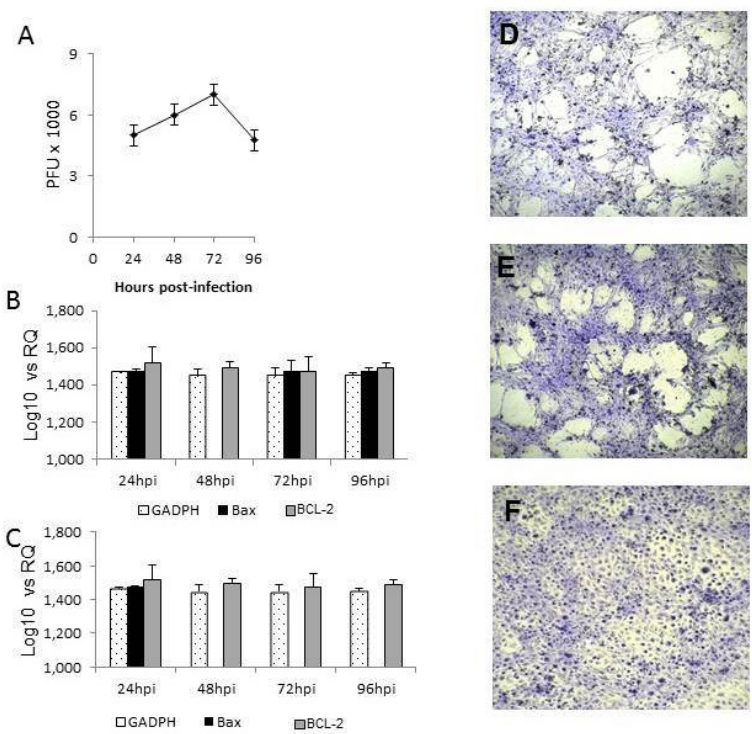

Figure 3. Kinetics of BoHV-5 replication and Bax/BCL-2 mRNA levels at different time points post-infection. The bWJ-MSCs infection revealed a productive infection (A) with plaque formation among infected monolayers (D and E) in comparison to uninfected ones (F). Real-time PCR was used to quantify the mRNA levels and fold-changes were calculated by $\Delta \Delta \mathrm{Ct}$ method as compared with non-infection cell control (B) and using endogeneous bovine GADH mRNA level to normalization. The infected cells completely suppress the Bax mRNA expression after 48 $\mathrm{h}$ of infection $(\mathrm{P}<0.001)$. 


\section{DISCUSSION}

This study was conducted to evaluate the impact of BoHV-5 infection and to explore the expression of BCL-2/Bax apoptotic mediators in this context. As primary cell cultures are relatively difficult to obtain and maintain over several passages, Madin-Darby bovine kidney cells (MDBK) are commonly used for BoHV-5 isolation and multiplication (Cardoso et al., 2007; Marin et al., 2012). Some $\alpha$-herpesviruses, such pseudorabies virus (PrV) and herpes simplex virus (HSV) are known to grow in a wide range of cell types, but the host range of BoHV-5 has not been extensively investigated (Deruelle et al., 2010; Esaki et al., 2010; Dufour et al., 2011). This study provides new information regarding BoHV-5 replication in bovine nonepithelial cells. During virus replication, bWJ-MSCs cultures developed cytopathologic effects similar to those reported for MDBK cells (Marin et al., 2012). Moreover, parameters related to the in vitro replication of several BoHV-5 isolates, such as the plaque size after infection of MDBK monolayers, have been reported by Marin et al. (2012). In comparison, plaque morphology and viral titers observed after BoHV-5 infection of bWJ-MSCs were similar.

Induction and suppression of apoptosis by viral infection are common events in viral pathogenesis (Griffin et al., 2010). BCL-2 has been localized to the nuclear membrane, endoplasmic reticulum and also the outer mitochondrial membrane (Ohta and Nishiyama, 2011). In addition, Bax is an integral organelle membrane protein, in particular in mitochondria (Ohta and Nishiyama, 2011). These two proteins are closely related to the balance of apoptosis and anti-apoptosis phenomena related to virus infection. In this study, in early stages of BoHV-5 replication (24-48 h post-infection), an increase in BCL-2 expression coincided with the increase in viral titer. This modulation has also been described for other members of the family Herpesviridae (Ohta and Nishiyama, 2011), but this is the first description related to BoHV-5. Based on the fact that over 130 distinct Herpesvirus species have been identified to date as a group, boasting an impressive broad host spectrum, the activation of some anti-apoptotic mediators to promote cell survival seems to be crucial for viral replication (Griffin et al., 2010). Mesenchymal stem cells (MSCs) are multipotent progenitor cells and they can be cultured from adult and fetal tissues (Cremonesi et al., 2011). Recently, a bovine mesenchymal cell line has been described, where it was isolated from Wharton's jelly layer (Cardoso et al., 2012). This biological characteristic of immune regulation has been demonstrated by others (Cremonesi et al., 2011). In conclusion, the use of MSCs in virology studies open new perspectives for future studies related to virus-cell interactions.

To our knowledge this is the first study of the role of mitochondria in BoHV-5 infection. BCL-2 release during in vitro BoHV-5 replication provides new information on the hostvirus interaction.

\section{ACKNOWLEDGMENTS}

Research supported by FAPESP (Grant \#2009/17635-3 and \#2010/50782-7) and CNPq (Brazilian Council for Research; T.C. Cardoso and E.F. Flores). We are grateful for the donation of the bovine umbilical cords.

\section{REFERENCES}

Cardoso TC, Ferrari HF, Luvizotto MCR and Arns CW (2007). Bio-safety technology in production of bovine herpesvirus type 5 (BoHV-5) using an alternative serum-free medium. Am. J. Biochem. Biotechnol. 3: 125-130. 
Cardoso TC, Ferrari HF, Garcia AF, Novais JB, et al. (2012). Isolation and characterization of Wharton's jelly-derived multipotent mesenchymal stromal cells obtained from bovine umbilical cord and maintained in a defined serum-free three-dimensional system. BMC Biotechnol. 12: 18.

Clarke P and Tyler KL (2009). Apoptosis in animal models of virus-induced disease. Nat. Rev. Microbiol. 7: 144-155.

Cremonesi F, Corradetti B and Lange CA (2011). Fetal adnexa derived stem cells from domestic animal: progress and perspectives. Theriogenology 75: 1400-1415.

Deruelle MJ, De Corte N, Englebienne J, Nauwynck HJ, et al. (2010). Pseudorabies virus US3-mediated inhibition of apoptosis does not affect infectious virus production. J. Gen. Virol. 91: 1127-1132.

Dufour F, Sasseville AM, Chabaud S, Massie B, et al. (2011). The ribonucleotide reductase R1 subunits of herpes simplex virus types 1 and 2 protect cells against TNF $\alpha$ - and FasL-induced apoptosis by interacting with caspase- 8 . Apoptosis 16: 256-271.

Esaki S, Goshima F, Katsumi S, Watanabe D, et al. (2010). Apoptosis induction after Herpes simplex virus infection differs according to cell type in vivo. Arch. Virol. 155: 1235-1245.

Ferrari HF, Luvizotto MC, Rahal P and Cardoso TC (2007). Detection of bovine herpesvirus type 5 in formalin-fixed, paraffin-embedded bovine brain by PCR: a useful adjunct to conventional tissue-based diagnostic test of bovine encephalitis. J. Virol. Methods 146: 335-340.

Griffin BD, Verweij MC and Wiertz EJ (2010). Herpesviruses and immunity: the art of evasion. Vet. Microbiol. 143: 89-100.

Marin MS, Leunda MR, Verna AE, Faverin C, et al. (2012). In vitro replication of bovine herpesvirus types 1 and 5. J. Virol. Methods 181: 80-85.

Ohta A and Nishiyama Y (2011). Mitochondria and viruses. Mitochondrion 11: 1-12.

West AP, Shadel GS and Ghosh S (2011). Mitochondria in innate immune responses. Nat. Rev. Immunol. 11: 389-402. 